\title{
Abugomry Induction Heater Design to Study the Thermal Properties of Magnetic Nanoparticles
}

\author{
Eltayeb Ibrahim Ahmed Elbeshir \\ Physics Department, Faculty of Science and Art, Almikhwah, Al-Baha University, KSA
}

\begin{abstract}
In this project a new simple induction heater design (Abugomry) operated at low power and low frequency $100 \mathrm{w} / 100 \mathrm{kHz}$ was made. The thermal properties of three different MNPs (magnetic nano particles) were studied by Abugomry induction heater. The high temperatures of MNPs $\left(47,46\right.$ and 50) ${ }^{\circ} \mathrm{C}$, the heating rate $(0.030,0.025$ and 0.028$){ }^{\circ} \mathrm{C} / \mathrm{min}$ and the specific absorption rate $\left(126,115\right.$ and 105) W/g for the $\left(\alpha-\mathrm{Fe}_{2} \mathrm{O}_{3}, \mathrm{Fe}_{3} \mathrm{O}_{4}\right.$ and $\left.\mathrm{CoFe}_{2} \mathrm{O}_{4}\right) \mathrm{MNPs}$ respectively, these results were suitable to use these MNPs in MHT (magnetic hyperthermia treatment). The results accrue from Abugomry of these MNPs that it's agree with the published results of the same MNPs, which were studied by induction heater operated at high/medium power and frequency.
\end{abstract}

Key words: Induction heater, thermal properties, magnetic nanoparticles, hyperthermia.

\section{Introduction}

The concepts of induction heating have been known and applied to manufacturing many years ago. The heat actually "induced" within the part itself by circulating electrical currents in this heating. While on the other hand in the most common heating methods, a torch or open flame is directly applied to the metal part. Induction heating depends on the RF (radio frequency) characteristics energy - that portion of the EMS (electromagnetic spectrum) below IR (infrared) and microwave energy. Since heat is transferred to the product via electromagnetic waves, the part never comes into direct contact with any flame, the inductor does not get hot, and there is no product contamination. When properly set up, the process becomes very repeatable and controllable $[1,2]$.

Induction heating is the process of heating an electrically conducting object (usually a metal) by electromagnetic induction, through heat generated in the object by eddy currents. An induction heater consists of an electromagnet, and an electronic oscillator that passes a high-frequency AC (alternating

Corresponding author: Eltayeb Ibrahim Ahmed Elbeshir, Dr., assistant professor, research field: physics of biomedical materials. current) through the electromagnet. The rapidly AMF (alternating magnetic field) penetrates the object, generating electric currents inside the conductor called eddy currents. The eddy currents flowing through the resistance of the material heat it by Joule heating. In magnetic and materials like iron, heat may also be generated by magnetic hysteresis losses $[1,3]$.

Induction heating allows the targeted heating of an applicable item for applications including surface hardening, melting, brazing and soldering and heating to fit. Iron and its alloys respond best to induction heating, due to their ferromagnetic nature $[1,2]$.

\section{Experimental Work}

\subsection{The Circuit and Components}

$\mathrm{R} 1=\mathrm{R} 2=27$ ohms, $5 \mathrm{~W}$; D1 = D2 = IN4007; T1 = $\mathrm{T} 2=100 \mathrm{~V}-35 \mathrm{~A}$, MOSFETs (IRFZ44n); $\mathrm{C}=0.75 \mu \mathrm{F}$ $-250 \mathrm{~V} ; \mathrm{L} 1=6-10$ terns of copper pipe or 10-14 terns of insolated one face wire with suitable diameter 4-6 $\mathrm{mm}$ as induction coil and $\mathrm{L} 2=2 \mathrm{mH}$ or 8 turns of 2 $\mathrm{mm}$ thick magnet wire on a toroidal ferrite core [2].

\subsection{Abugomry Induction Heater Setup}

(1) Two induction coils were made the first one by 10-14 turns of insolated wire in 4-6 mm diameter in 
Fig. 2a. While the second by 6-10 turns of Copper pipe in 4-6 mm diameter is shown in Fig. 2b;

(2) The circuit of induction heating was connected and tested in the board by using DC power supply 0-24 $\mathrm{V}$ and $5 \mathrm{~A}$ maximum. The multimeter was used in the frequency function as Figs. $2 \mathrm{c}$ and $2 \mathrm{~d}$ show;

(3) The circuit established on the secondhand computer's power supply box because the fan was used to cool down the circuit and the circuit cheek after that (Figs. 2e and 2f);

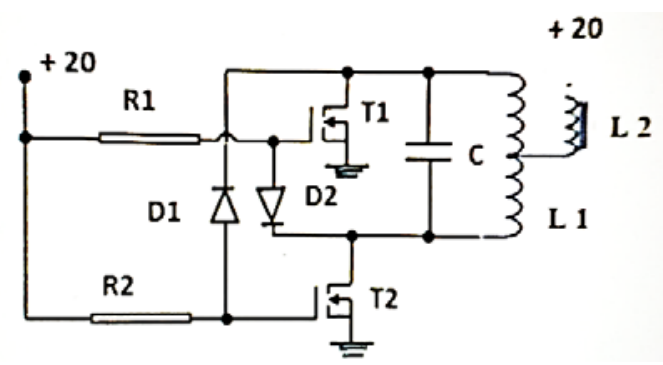

Fig. 1 The circuit of Abugomry induction heater.

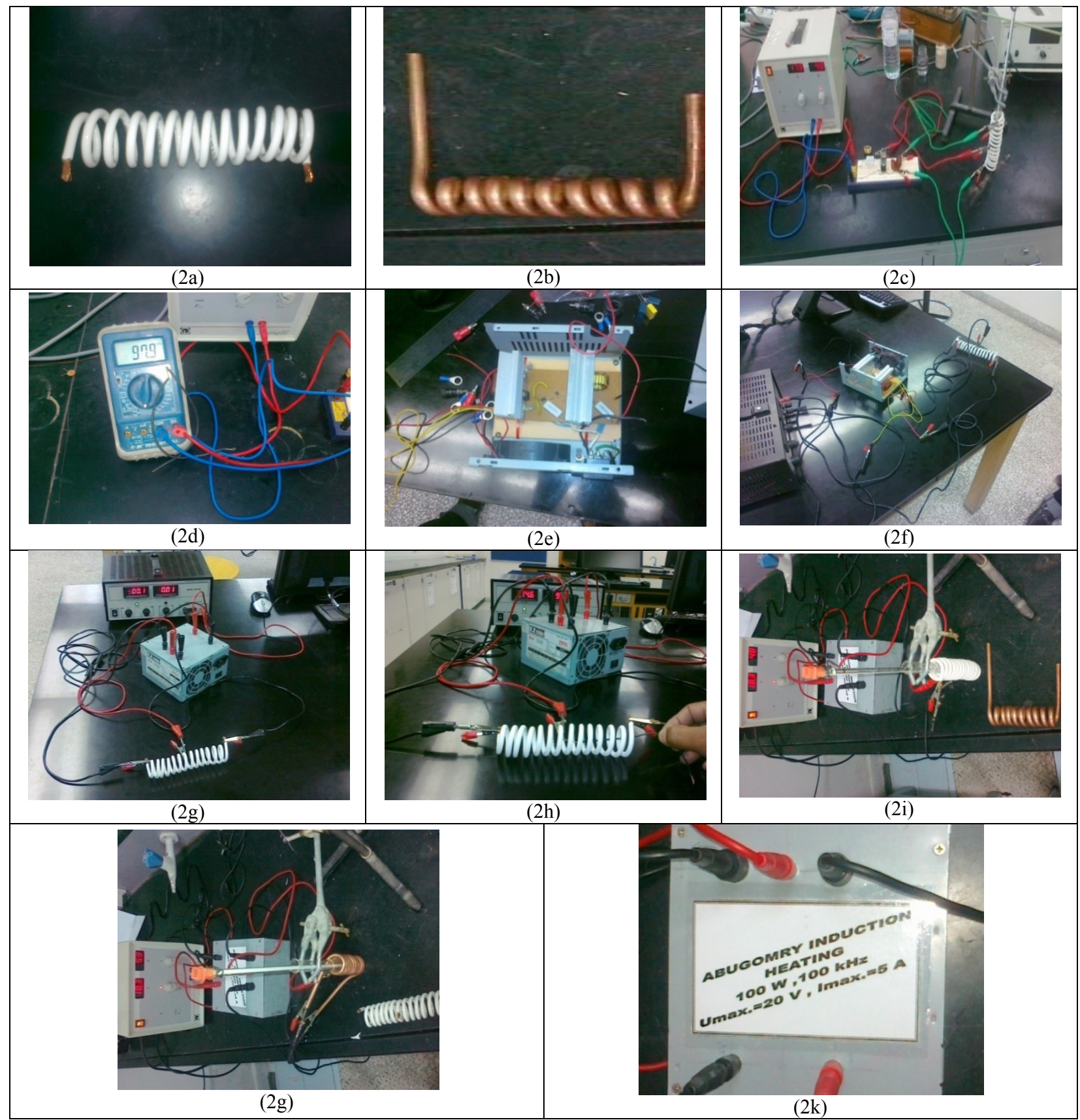

Fig. 2 Abugomry induction heater setup. 
(4) Test the circuit after established on the secondhand computer's power supply box (Figs. $2 \mathrm{~g}$ and $2 \mathrm{~h}$ );

(5) Test the circuit after closed the secondhand computer's power supply box (Figs. $2 \mathrm{i}$ and $2 \mathrm{j}$ );

(6) In Fig. $2 \mathrm{k}$ the box was closed to give a good design of the induction heating called Abugomry and connecting to cheek it by magnetic material.

\subsection{The Suspensions of MNPs}

To study the thermal properties of $\left(\alpha-\mathrm{Fe}_{2} \mathrm{O}_{3}\right.$, $\mathrm{Fe}_{3} \mathrm{O}_{4}$ and $\left.\mathrm{CoFe}_{2} \mathrm{O}_{4}\right)$ MNPs, three suspensions were made, any one contain $1 \mathrm{mg}$ of MNPs $+1 \mathrm{ml}$ of DW (distil water) respectively[4].

\section{Results and Discussion}

Abugomry induction heater made above was used to study the thermal properties of three samples of $\left(\alpha-\mathrm{Fe}_{2} \mathrm{O}_{3}, \mathrm{Fe}_{3} \mathrm{O}_{4}\right.$ and $\left.\mathrm{CoFe}_{2} \mathrm{O}_{4}\right)$ MNPs.

The temperature time curves of these samples were shown in Figs. 3, 4 and 5 respectively. They were published in RJSITM, IJSR and RJSITM respectively [4-6].

There are many factors affecting on the thermal properties of MNPs such as the type and size of MNPs itself, the time of heating, the frequency and intensity of magnetic field was applied on the nano particles and the power also [7-10].

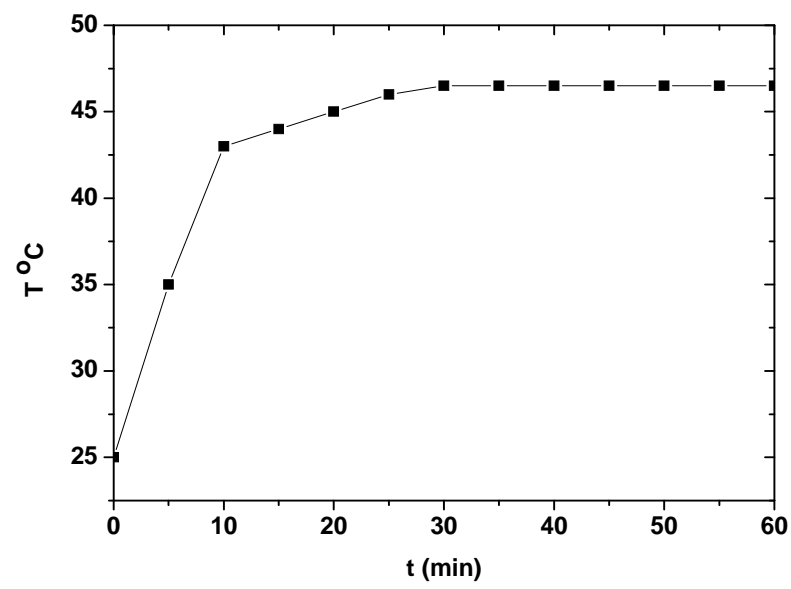

Fig. $3 \alpha-\mathrm{Fe}_{2} \mathrm{O}_{3}$ heating rate [4].

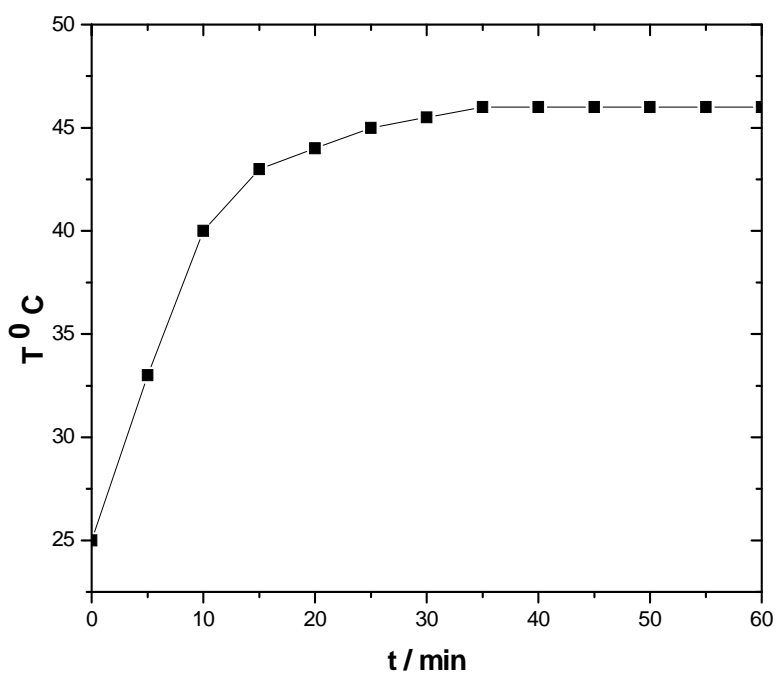

Fig. $4 \mathrm{Fe}_{3} \mathrm{O}_{4}$ heating rate [5].

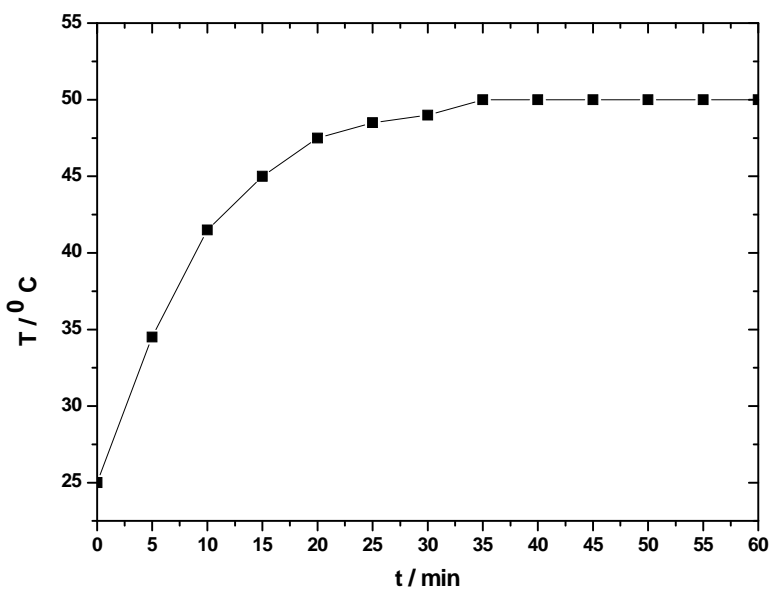

Fig. $5 \mathrm{CoFe}_{2} \mathrm{O}_{4}$ heating rate [6].

Table 1 MNPs size, temperature, heating rate and specific absorption rate.

\begin{tabular}{lllll}
\hline MNPs & $\mathrm{t}$ & $\mathrm{T}$ & $\Delta \mathrm{T} / \Delta \mathrm{t}$ & $\mathrm{SAR}$ \\
& $\mathrm{nm}$ & $\begin{array}{l}{ }^{\circ} \mathrm{C} \\
\mathrm{o} / \mathrm{g}\end{array}$ \\
\hline$\alpha-\mathrm{Fe}_{2} \mathrm{O}_{3}$ & 64 & 47 & 0.030 & 126 \\
$\mathrm{Fe}_{3} \mathrm{O}_{4}$ & 10 & 46 & 0.025 & 105 \\
$\mathrm{CoFe}_{2} \mathrm{O}_{4}$ & 13 & 50 & 0.028 & 115 \\
\hline
\end{tabular}

The size, the maximum temperatures, the heating rate $\Delta \mathrm{T} / \Delta \mathrm{t}$ and the specific absorption rate $\mathrm{SAR}$ of these MNPs $\left(\alpha-\mathrm{Fe}_{2} \mathrm{O}_{3}, \mathrm{Fe}_{3} \mathrm{O}_{4}\right.$ and $\left.\mathrm{CoFe}_{2} \mathrm{O}_{4}\right)$ shown in Table 1 was published in IJSR [11].

After we compared the results accrue from Abugomry induction heater operating at $100 \mathrm{~W} / 100 \mathrm{kHz}$, with the results were published in [12-16] we find that Abugomry induction heater is 
quite suitable to study the thermal properties of MNPs.

\section{Conclusion}

(1) The thermal properties of MNPs were studied by Abugomry induction heater agree with the published results of the same MNPs were studied by induction heater operated at high/medium power and frequency;

(2) Abugomry induction heater operated at law power and frequency $100 \mathrm{~W} / 100 \mathrm{kHz}$, while the researchers used induction heaters operated at high or medium power and frequency;

(3) Abugomry induction heater is very cheap comparative with anther induction heaters and any researcher can make it in fundamental physics laboratory;

(4) The disadvantage of Abugomry induction heater was operated at fixed frequency $100 \mathrm{kHz}$ only.

\section{References}

[1] https://en.wikipedia.org/wiki/Induction_heating.

[2] www.rmcybernetics.com/projects/DIY.../diy-induction-he ater.htm.

[3] www.richieburnett.co.uk/indheat.html.

[4] Elbeshir, E. I. A. 2015. "The Thermal Properties and the Energy Absorption of $\alpha-\mathrm{Fe}_{2} \mathrm{O}_{3}$ Nanoparticles at Low Frequency." RJSITM 4 (07) Ma.

[5] Elbeshir, E. I. A. 2015. "Synthesis and Specific Absorption Rate of $\mathrm{Fe}_{3} \mathrm{O}_{4}$ Nanoparticles." IJSR 4 (2) February.

[6] Elbeshir, E. I. A., Yassin, O. A., and Khalil A. Z. 2013. "Study of $\mathrm{CoFe}_{2} \mathrm{O}_{4}$ Nano-Particles Energy Absorption Used a New Induction Heating Design Operating at 100W-100 kHz.” RJSITM 02 (05) March.
[7] Tomitaka, A., Jeun, M., Bae, S., and Takemura, Y. 2011. "Evaluation of Magnetic and Thermal Properties of Ferrite Nanoparticles for Biomedical Applications." Journal of Magnetics 16 (2):164-8. DOI:10.4283/JMAG.

[8] Sriharsha, T., and Bahadur, D. 2005. "Preparation and Investigation of Potentiality of Different Soft Ferrites for Hyperthermia Applications.” Journal of Applied Physics 97: 10Q916s, d.

[9] Suto, M., Hirota, Y., Mamiya, H., Fujita, A., Kasuya, R., Tohji, K., and Jeyadevan, B. 2009. "Heat Dissipation Mechanism of Magnetite Nano Particles in Magnetic Fluid Hyperthermia." Journal of Magnetism and Magnetic Materials 321: 1493-6.

[10] Zhang, X. K., Li, Y. F., and Xiaoa, J. Q. 2003. "Theoretical and Experimental Analysis of Magnetic Inductive Heating in Ferrite Materials." Journal of Applied Physics 93 (1015) May.

[11] Elbeshir, E. I. A. 2016. "Evaluation of Thermal Properties of Ferrite Nanoparticles for Magnetic Hyperthermia Treatment." IJSR 5 (2) February.

[12] Skumiel, A., and Łabowski, M. 2006. "The Heating Effect of the Biocompatible Ferro Fluid in Alternating Magnetic Field." Molecular and Quantum Acoustics 27: 233.

[13] Motoyama, J., Hakata, T., Kato, R., Yamashita, N., Morino, O., Kobayashi, T., and Honda, H. 2008. "Size Dependent Heat Generation of Magnetite Nanoparticles under AC Magnetic Field for Cancer Therapy." Bio Magnetic Research and Technology pen Access.

[14] Ma, M., Wu, Y., Zhou, J., Sun, Y. K., Zhang, Y., and Gu, N. 2004. "Size Dependence of Specific Power Absorption of $\mathrm{Fe}_{3} \mathrm{O}_{4}$ Particles in AC Magnetic Field." Journal of Magnetism and Magnetic Materials 268: 33-9.

[15] Ramanujan, R. V., Ang, K. L., and Venkatraman, S. 2009. "Magnet-PNIPA hydrogels for bioengineering applications." J. Mater. Sci. 44: 1381-7.

[16] Chenab, Y., Ruana, M., Jianga, Y. F., Chengb, S. G., and Lia, W. 2010. "The Synthesis and Thermal Effect of $\mathrm{CoFe}_{2} \mathrm{O}_{4}$ Nanoparticles." Journal of Alloys and Compounds 493: L36-8. 